\title{
Averaging Effects on Irregularities in the Distribution of Primes in Arithmetic Progressions
}

\section{By Richard H. Hudson}

Abstract. Let $t$ be an integer taking on values between 1 and $x\left(x\right.$ real), let $\pi_{b, c}(t)$ denote the number of positive primes $\leqslant t$ which are $\equiv c(\bmod b)$, and let li $t$ denote the usual integral $\operatorname{logarithm}$ of $t$. Further, let the ratio of quadratic nonresidues of $b>2$ to quadratic residues of $b$ be $\gamma(b)$ to 1 , and let

$$
A_{h}(x)=(1 / \gamma(b)) \frac{1}{x}\left(\sum_{\substack{t=1 \\ 1 \leqslant c \leqslant b-1}}^{x} \pi_{h, c}(t)-\gamma(b) \sum_{\substack{t=1 \\ 1 \leqslant c^{\prime} \leqslant b-1}}^{x} \pi_{h, c^{\prime}}(t)\right)
$$

where $c$ runs over quadratic nonresidues and $c^{\prime}$ runs over quadratic residues of $b$.

Nearly periodic oscillations of $A_{6}(x)=(1 / x) \sum_{t=1}^{x}\left(\pi_{6.5}(t)-\pi_{6.1}(t)\right)$ about $h(x)=$ $(1 / x) \sum_{t=1}^{x} \mathrm{li}\left(t^{1 / 2}\right) / 2$ are depicted in Figures 2, 3, 4 over the range of integers less than $2.5 \times 10^{11}$. Over this range, $h(x)$ is a far better "axis of symmetry" for these oscillations than $s(x)=(1 / x) \sum_{t=1}^{x} t^{1 / 2} / \log t$ (suggested by Shanks [29]).

On the other hand, recent work of W. J. Ellison [9], three letters from Andrzej Schinzel to the author, and my own considerations (see Section 4) lead to the following. In contradiction to a conjecture of Shanks [29],

$$
\frac{1}{x}\left(\sum_{t=1}^{x}\left(\pi_{6.5}(t)-\pi_{6.1}(t)\right) /\left(t^{1 / 2} / \log t\right)\right) \nrightarrow 1 \text { as } x \rightarrow \infty .
$$

Moreover, I prove in Theorem 4.1 that $A_{6}(x) / h(x) \nrightarrow 1$ as $x \rightarrow \infty$, and Schinzel has provided a heuristic argument that no amount of averaging of $A_{6}(x)$ will provide an asymptotic relationship of this sort. However, let $h^{(1)}(x)=h(x), A_{6}^{(1)}(x)=A_{6}(x)$, and for $k>1$ let

$$
h^{(k+1)}(x)=\frac{1}{x} \sum_{t=1}^{x} h^{(k)}(t), \quad A_{6}^{(k+1)}(x)=\frac{1}{x} \sum_{t=1}^{x} A_{6}^{(k)}(t) .
$$

Assuming the truth of the generalized Riemann hypothesis for $L(s, \chi), \chi$ the nonprincipal character $\bmod 6$, we prove

$$
\lim _{k \rightarrow \infty} \varlimsup_{x \rightarrow \infty} \frac{A_{6}^{(k)}(x)}{h^{(k)}(x)}=1=\lim _{k \rightarrow \infty} \lim _{x \rightarrow \infty} \frac{A_{6}^{(k)}(x)}{h^{(k)}(x)} .
$$

The behavior of $A_{6}(x)$ is a special case of a far more general phenomenon. In Section 3, reasons are given why $A_{h}(x)$ can be expected to oscillate more or less symmetrically about $h(x)$ for every modulus $b>2$.

1. Introduction and Summary. Throughout $b$ will denote a modulus $>2$; $c$ will always denote a quadratic nonresidue and $c^{\prime}$ a quadratic residue of $b$. Let $t$ be an integer with $1 \leqslant t \leqslant x(x$ real $)$ and let $\pi_{b, c}(t)$ and $\pi_{b, c^{\prime}}(t)$ denote, respectively, the

Received December 29, 1979.

1980 Mathematics Subject Classification. Primary 10H15, 10H20, 10-04; Secondary 10H05. 
number of primes $\leqslant t$ which are $\equiv c(\bmod b)$ or to $c^{\prime}(\bmod b)$. For each $b=2^{\alpha_{0}} q_{1}^{\alpha_{1}}$ $\cdots q_{r}^{\alpha_{r}}, q_{1}, \ldots, q_{r}$ distinct odd primes, it is well-known (see, e.g., [26]) that the ratio of quadratic nonresidues to residues is $2^{r+\beta-1}-1$ to 1 where $\beta=1$ if $\alpha_{0}=0$ or 1 , $\beta=2$ if $\alpha_{0}=2$, and $\beta=3$ if $\alpha_{0} \geqslant 3$. Consequently, one would expect that

$$
A_{b}(x)=\frac{1}{\left(2^{r+\beta-1}-1\right) x} \sum_{\substack{t=1 \\ 1 \leqslant c \leqslant b-1}}^{x} \pi_{b, c}(t)-\frac{1}{x} \sum_{\substack{t=1 \\ 1 \leqslant c^{\prime} \leqslant b-1}}^{x} \pi_{b, c^{\prime}}(t)
$$

would oscillate more or less evenly about 0 . Daniel Shanks [29] first observed that for $x<3.10^{6}$, and certain values of $b$, this is not the case.

Figures 2 and 3 at the conclusion of this paper depict oscillations of

$$
A_{6}(x)=\frac{1}{x} \sum_{t=1}^{x}\left(\pi_{6,5}(t)-\pi_{6,1}(t)\right)
$$

about

$$
h(x)=\frac{1}{x} \sum_{t=1}^{x} \frac{\text { li } t^{1 / 2}}{2}
$$

on standard scales and in Figure 4 they are depicted for $x<250,000,000,000$ on a logarithmic scale. These figures show clearly that $h(x)$ is an excellent "axis of symmetry" for these nearly periodic oscillations. Indeed, over this considerable range, the approximation (1.2) is markedly superior to $s(x)=(1 / x) \sum_{t=1}^{x} t^{1 / 2} / \log t$ although $h(x)$ and $s(x)$ are asymptotically equal (as we will see $h(x)-s(x) \sim$ $\left.(4 / 3) x^{1 / 2} / \log ^{2} x\right)$.

In Section 4 we show that averaging in the ordinary sense (as in (1.1) and (1.2)) only postpones the swamping of the Chebyshev phenomenon by the giant fluctuations discovered by Hardy and Littlewood (see [16]).

In particular, with the help of A. Schinzel we prove

THEOREM 4.1. It is false that

$$
\lim _{x \rightarrow \infty} \frac{A_{6}(x)}{h(x)}=1
$$

Moreover, we give a heuristic argument that no amount of ordinary averaging will yield such a limit. This is interesting as it suggests that the Abelian averaging employed by, e.g., Knapowski and Turán [17]-[22] is in a sense stronger than ordinary averaging. Of course, the results of Knapowski and Turán are contingent on the truth of the generalized Riemann hypothesis as is our

THEOREM 4.2. Let $A_{6}^{(k)}(x)$ and $h^{(k)}(x)$ denote the kth averaging of $A_{6}(x)$ and $h(x)$. Then

$$
\lim _{k \rightarrow \infty} \varlimsup_{x \rightarrow \infty} \frac{A_{6}^{(k)}(x)}{h^{(k)}(x)}=1=\lim _{k \rightarrow \infty} \frac{\lim }{x \rightarrow \infty} \frac{A_{6}^{(k)}(x)}{h^{(k)}(x)}
$$

The nearly periodic behavior of the oscillations of $A_{6}(x)$ about $h(x)$ is interesting and should be compared with the important works of Pólya [27], Bloch and Pólya [5], and Grosswald [10]. 
2. Preliminaries-Computation of $s(x)$ and $h(x)$. Integration by parts and a straightforward treatment of the error term (see, e.g., [1]), yields for each fixed $k_{1}$,

$$
\begin{aligned}
s(x)= & \frac{1}{x} \sum_{t=1}^{x} t^{1 / 2} / \log t=\frac{2 x^{1 / 2}}{3 \log x}+\frac{4 x^{1 / 2}}{9 \log ^{2} x}+\frac{16 x^{1 / 2}}{27 \log ^{3} x} \\
& +\frac{32 x^{1 / 2}}{27 \log ^{4} x}+\cdots+\frac{\left(\frac{2}{3}\right)^{k_{1}}\left(k_{1}-1\right) ! x^{1 / 2}}{\log ^{k_{1}} x}+\mathcal{O} \frac{x^{1 / 2}}{\log ^{k_{1}+1} x} .
\end{aligned}
$$

Our computer program utilized a simple trapezoidal rule. Five terms of the above expansions are sufficient for a high level of accuracy.

Moreover,

$$
\begin{aligned}
\frac{\operatorname{li} x^{1 / 2}}{2}= & \frac{x^{1 / 2}}{\log x}+\frac{2 x^{1 / 2}}{\log ^{2} x}+\frac{8 x^{1 / 2}}{\log ^{3} x}+\frac{48 x^{1 / 2}}{\log ^{4} x} \\
& +\cdots+\frac{2^{k_{2}-1}\left(k_{2}-1\right) ! x^{1 / 2}}{\log ^{k_{2}} x}+\mathcal{O}\left(\frac{x^{1 / 2}}{\log ^{k_{2}+1} x}\right) .
\end{aligned}
$$

Consequently, we may integrate by parts to obtain for each fixed $k_{3}$,

$$
\begin{aligned}
h(x) \sim & \lim _{\eta \rightarrow 0^{+}} \frac{1}{x}\left(\left(\int_{0}^{1-\eta}+\int_{1+\eta}^{x}\right) \frac{\operatorname{li} t^{1 / 2}}{2} d t\right) \\
= & \frac{2 x^{1 / 2}}{3 \log x}+\frac{16 x^{1 / 2}}{9 \log ^{2} x}+\frac{208 x^{1 / 2}}{27 \log ^{3} x}+\frac{1280 x^{1 / 2}}{27 \log ^{4} x}+\frac{30976 x^{1 / 2}}{81 \log ^{5} x} \\
& +\cdots+\frac{2^{k_{3}}\left(k_{3}-1\right) !\left(1+3+9+\cdots+3^{k_{3}-1}\right) x^{1 / 2}}{3^{k_{3}} \log ^{k_{3}} x}+\mathcal{O}\left(\frac{x^{1 / 2}}{\log ^{k_{3}+1} x}\right) \\
= & \sum_{j=1}^{k_{3}} \frac{2^{j-1}(j-1) !\left(3^{j}-1\right) x^{1 / 2}}{3^{j} \log ^{j} x}+\mathcal{O}\left(\frac{x^{1 / 2}}{\log ^{k_{3}+1} x}\right) .
\end{aligned}
$$

The reader may find it easier to verify (2.3) by differentiation than to derive it by integration. The implied constant in (2.3) is significantly larger than in (2.1). Consequently, the most serious error present in Figures 2, 3, 4 arises from the failure to take $k_{3}>5$ in using (2.3). However, even at $10^{11}$, the use of seven or eight terms would raise the curve representing $h(x)$ by an almost imperceptible amount.

Let $h^{(2)}(x)=(1 / x) \sum_{t=1}^{x} h(t)$. By means of a second integration by parts we have for each fixed $k_{4}$,

$$
\begin{aligned}
h^{(2)}(x) \sim & \frac{4 x^{1 / 2}}{9 \log x}+\frac{40 x^{1 / 2}}{27 \log ^{2} x}+\frac{576 x^{1 / 2}}{81 \log ^{3} x}+\frac{11136 x^{1 / 2}}{243 \log ^{4} x}+\cdots \\
& +\frac{2^{k_{4}+1}\left(k_{4}-1\right) !\left(1+1+3+1+3+9+\cdots+1+3+9+\cdots+3^{k_{4}-1}\right) x^{1 / 2}}{3^{k_{4}+1} \log ^{k_{4}} x} \\
& +\mathcal{O}\left(\frac{x^{1 / 2}}{\log ^{k_{4}+1} x}\right) \\
= & \sum_{j=1}^{k_{4}} \frac{2^{j-1}(j-1) !\left(3^{j+1}-2 j-3\right) x^{1 / 2}}{3^{j+1} \log ^{j} x}+\mathcal{O}\left(\frac{x^{1 / 2}}{\log ^{k_{4}+1} x}\right) .
\end{aligned}
$$


Remark. The numerically inclined reader may be interested in plotting $(1 / x) \sum_{t=1}^{x} A_{6}(t)$ versus $h^{(2)}(x)$ for his own curiosity. In doing so, one might choose to use (over a small range)

$$
\text { li } x=\lim \left(\int_{0}^{1-\eta}+\int_{1-\eta}^{x}\right) \frac{d u}{\log u}=\gamma+\log \log x+\sum_{k=1}^{\infty} \frac{(\log x)^{k}}{k ! k}
$$

in preference to the asymptotic expansion for li $x$;

$$
\gamma=\lim _{N \rightarrow \infty} \sum_{n=1}^{N} \frac{1}{n}-\log N \approx .577 .
$$

However, good convergence for this expansion at, say $10^{11}$, requires nearly 80 terms.

3. The Isolation of the "Negative Part" of $A_{b}(x)$. An excellent account of the standard argument for the modulus 4 may be found in Ingham [16, pp. 106-107] (although the replacement of $\pi\left(x^{1 / 2}\right) / 2$ by $x^{1 / 2} / \log x$ on p. 107 is unnecessary).

Recently, the author has given a purely elementary argument [14] which suggests that $\pi_{4,3}(x)-\pi_{4,1}(x)$ oscillates about $\pi\left(x^{1 / 2}\right) / 2$ at least for small $x$. A generalization (see [14]) of this argument motivates what follows.

Let $b=2^{\alpha_{0}} q_{1}^{\alpha_{1}} q_{2}^{\alpha_{2}} \ldots q_{r}^{\alpha_{r}}$ where $q_{1}, \ldots, q_{r}$ are distinct odd primes. The ratio of quadratic nonresidues of $b$ to quadratic residues of $b$ is $2^{r+\beta-1}-1$ to 1 where $\beta=1$ if $\alpha_{0}=0$ or $1, \beta=2$ if $\alpha_{0}=2$, and $\beta=3$ if $\alpha_{0} \geqslant 3$ (see, e.g., [26, p. 167]). With this in mind, we define

$$
\tau_{b}(s)= \begin{cases}2^{r+\beta-1}-1 & \text { if } s \text { is a quadratic residue of } b \\ -1 & \text { if } s \text { is a quadratic nonresidue of } b \\ 0 & \text { otherwise }\end{cases}
$$

Moreover, we define analogues of $\pi(x)$ and $\Pi(x)$ by

$$
\pi\left(x, \tau_{b}\right)=\sum_{p \leqslant x} \tau_{b}(p)
$$

where $p$ is prime, and

$$
\Pi\left(x, \tau_{b}\right)=\sum_{p^{m} \leqslant x} \frac{\tau_{b}\left(p^{m}\right)}{m} .
$$

In the following theorem we let $c^{\prime}$ denote a quadratic residue of $b$ and $c$ a quadratic nonresidue of $b$.

THEOREM 3.1. Let $b=2^{\alpha_{0}} \cdot q_{1}^{\alpha_{1}} \cdot q_{2}^{\alpha_{2}} \cdot q_{3}^{\alpha_{3}} \cdots q_{r}^{\alpha_{r}}$, where $b>2$ and $q_{1}, \ldots, q_{r}$ are distinct odd primes. Then,

$$
\begin{aligned}
\sum_{1 \leqslant c^{\prime} \leqslant b-1} \pi_{b, c^{\prime}}(x) & -\frac{1}{2^{r+\beta-1}-1}\left(\sum_{1 \leqslant c \leqslant b-1} \pi_{b, c}(x)\right) \\
& =\frac{\pi\left(x^{1 / 2}\right)}{2}\left[\left(-1+\frac{2}{2^{r+\beta-1}-1}\left(\frac{\Pi\left(x, \tau_{b}\right)}{\pi\left(x^{1 / 2}\right)}\right)\right)+o(1)\right] .
\end{aligned}
$$


Proof. Note that

$$
\pi\left(x, \tau_{b}\right)=\left(2^{r+\beta-1}-1\right) \sum_{1 \leqslant c^{\prime} \leqslant b-1} \pi_{b, c^{\prime}}(x)-\sum_{1 \leqslant c \leqslant b-1} \pi_{b, c}(x) .
$$

Moreover, since $\tau_{b}\left(p^{2}\right)=+1$ for every prime $p$ with $(b, p)=1$, we have (see $p$. 107 of [16])

$$
\Pi\left(x, \tau_{b}\right)-\pi\left(x, \tau_{b}\right)=\left(\frac{\left(2^{r+\beta-1}-1\right) \pi\left(x^{1 / 2}\right)}{2}\right)+\mathcal{O}\left(x^{1 / 3} \log x\right) .
$$

Now (3.4) follows easily from (3.5) and (3.6).

The extent to which (3.2) and (3.3) are natural analogues of $\pi(x)$ and $\Pi(x)$, respectively, is readily seen by noting that Theorem 3.1 reduces to the classical result in Ingham [16] if $b=4$ (or $b=6$ ). Specifically, if $b=4$ then $\tau_{b}$ is the real nonprincipal Dirichlet character, $\chi_{1}$, and $\Pi\left(x, \tau_{b}\right)$ is the function "naturally associated with $\log L\left(s, \chi_{1}\right)$ "'. As such, $\Pi\left(x, \chi_{1}\right)$ can be expected to have values fairly evenly distributed about zero. Consequently, $-\pi\left(x^{1 / 2}\right) / 2$ is called by Ingham the "negative part" and $\Pi\left(x, \chi_{1}\right)$ the "oscillating part" of (3.4) when $b=4$.

Let $b>2$ be a modulus admitting a primitive root, i.e., $b=4, q^{\alpha}$, or $2 q^{\alpha}$, where $q$ is an odd prime. Then $\tau_{b}$ is the real nonprincipal Dirichlet character (or Kronecker symbol) and the above argument is seen to be a natural special case.

When $b$ does not admit a primitive root, $2^{r+\beta-1}-1>1$. The resultant awkwardness in the appearance of (3.1) is (regrettably) necessary if one wishes to extend the above argument to arbitrary modulus. The reason that I find most compelling for feeling that for every $b$ the isolation of the term,

$$
\frac{-\pi\left(x^{1 / 2}\right)}{2}(1+o(1))
$$

in (3.4) is meaningful (at least for small $x$ ), is the fact that the elementary theory in Section 6 of [14] (see (6.14) of [14]) suggests that

$$
\frac{1}{2^{r+\beta-1}-1}\left(\sum_{1 \leqslant c \leqslant b-1} \pi_{h, c}(t)\right)-\sum_{1 \leqslant c^{\prime} \leqslant h-1} \pi_{b, c^{\prime}}(t)
$$

oscillates about $\left(\pi\left(t^{1 / 2}\right) / 2\right)(1+o(1))$.

Of course, if $b=4$ or 6 , the result of Littlewood [25] shows that the "negative part" is overcome infinitely often. (The case for general moduli is far from solved; for an interesting recent result, see Stark [32].) Moreover, the results of Ellison [9] and Theorem 4.1 of this paper show that (for $b=4$ or 6 ), even in the mean, the "oscillatory part" is not negligible relative to the "negative part."

4. Limitations of ordinary averaging. Recently W. J. Ellison [9] has disproven the conjecture of Shanks [29] that

$$
\lim _{x \rightarrow \infty} \frac{1}{x} \sum_{t=1}^{x} \frac{\pi_{4,3}(t)-\pi_{4,1}(t)}{t^{1 / 2} / \log t}=1 .
$$

A modification of this argument suggested to me by A. Schinzel (personal communication, May 30, 1977) yields the following theorem. 
THEOREM 4.1. It is false that

$$
\lim _{x \rightarrow \infty} \frac{A_{6}(x)}{h(x)}=1
$$

Proof. Let $\chi(n)=(n / 3)$ and

$$
L(s, \chi)=\sum_{n=1}^{\infty} \frac{\chi(n)}{n^{s}}, \quad \Pi(x, \chi)=\sum_{p^{m} \leqslant x} \frac{\pi\left(p^{m}\right)}{m}
$$

so that

$$
\Pi(t, \chi)-\pi(t, \chi)=\frac{\pi\left(t^{1 / 2}\right)}{2}+\mathcal{O}\left(t^{1 / 3} \log t\right) .
$$

Assume that $A_{6}(x) / h(x) \rightarrow 1$ as $x \rightarrow \infty$.

If $x$ is an integer, we have

$$
\begin{aligned}
\int_{1}^{x} \Pi(t, \chi) d t & =\int_{1}^{x} \pi(t, \chi) d t+\int_{1}^{x} \frac{\pi\left(t^{1 / 2}\right)}{2} d t+\mathcal{O}\left(\int_{1}^{x} t^{1 / 3} \log t d t\right) \\
& =\sum_{t=1}^{x-1} \pi\left(t_{1}, \chi\right)+\sum_{t=1}^{x-1} \frac{\pi\left(t^{1 / 2}\right)}{2}+\mathcal{O}\left(x^{4 / 3} \log x\right) \\
& =-(x-1) A_{6}(x-1)+(x-1) h(x-1)+\mathcal{O}\left(x^{4 / 3} \log x\right) .
\end{aligned}
$$

But, because of our assumption,

$$
-A_{6}(x-1)+h(x-1)=o(h(x-1))=o\left(x^{1 / 2} / \log x\right)
$$

so that

$$
-(x-1) A_{6}(x-1)+(x-1) h(x-1)=o\left(x^{3 / 2} / \log x\right) .
$$

If $x$ is not an integer,

$$
\int_{1}^{x} \Pi(t, \chi) d t=\int_{1}^{[x]} \Pi(t, \chi) d t+\mathcal{O}(\Pi(x, \chi)),
$$

and this is

$$
=o\left(x^{3 / 2} / \log x\right)+\mathcal{O}(x / \log x)=o\left(x^{3 / 2} / \log x\right) .
$$

Thus, in any case,

$$
\Pi_{1}(x, \chi)=\int_{1}^{x} \Pi(t, \chi) d t=o\left(x^{3 / 2} / \log x\right) .
$$

On the other hand, for $\operatorname{Re} s>1$,

$$
\begin{aligned}
\log L(s, \chi) & =s \int_{1}^{\infty} \frac{\Pi(x, \chi)}{x^{s+1}} d x \\
& =s \lim _{y \rightarrow \infty} \frac{\Pi_{1}(y, \chi)}{y^{s+1}}+(s+1) \int_{1}^{y} \frac{\Pi_{1}(x, \chi)}{x^{s+2}} d x \\
& =s(s+1) \int_{1}^{\infty} \frac{\Pi_{1}(x, \chi)}{x^{s+2}} d x
\end{aligned}
$$

see (6.23) of [9]. 
Now (4.10) implies the convergence of the integral on the right-hand side for $\operatorname{Re} s>\frac{1}{2}$, hence the nonvanishing of $L(s, \chi)$ to the right of $\sigma=\frac{1}{2}$. Consider the function

$$
\begin{aligned}
f(s) & =-\frac{d}{d s}\left(\frac{\log L(s, \chi)}{s(s+1)}\right) \\
& =\frac{-1}{s(s+1)} \frac{L^{\prime}(s, \chi)}{L(s, \chi)}+\frac{2 s+1}{s^{2}(s+1)^{2}} \log L(s, \chi) .
\end{aligned}
$$

By (4.11), we have

$$
f(s)=\frac{-d}{d s} \int_{1}^{\infty} \frac{\Pi_{1}(x, \chi)}{x^{s+2}} d x=\int_{1}^{\infty} \frac{\Pi_{1}(x, \chi) \log x}{x^{s+2}} d x .
$$

If now, $s=\sigma+i t, \sigma \rightarrow(1 / 2)^{+}$, and (4.10) holds, we have

$$
|f(x)|=o\left(\int_{1}^{\infty} \frac{x^{3 / 2}}{\left|x^{s+2}\right|} d x\right)=o\left(\frac{1}{\sigma-\frac{1}{2}}\right),
$$

a contradiction, since then $L(s, \chi)$ has no zero on the line $\sigma=\frac{1}{2}$ !

Using the Riemann $\zeta$ function it is easy to modify the above to show the falsity of Shanks' conjecture [29] that

$$
\lim _{x \rightarrow \infty} \frac{1}{x} \sum_{t=1}^{x} \frac{\operatorname{li} t-\pi(t)}{t^{1 / 2} / \log t}=1 .
$$

The well-known result of Hardy and Littlewood [11] and Landau [23], [24], rephrased as on p. 276 of [29], states that there exists a positive constant $k$ with

$$
A_{6}(x)>k x^{1 / 2} / \log x
$$

if and only if the generalized Riemann hypothesis is true for $L(s, \chi), \chi$ the nonprincipal character mod6. If (4.2) were true, one would have the very much stronger result,

$$
A_{6}(x) \sim 2 x^{1 / 2} / 3 \log x \quad(\text { as } x \rightarrow \infty) .
$$

Seen in this light the falsity of (4.1) is not entirely surprising in spite of the relatively good behavior of $A_{6}(x)$ over the first 250 billion integers.

Indeed, Schinzel has communicated the following heuristic argument that no amount of averaging will yield an asymptotic relationship of this sort. In particular, letting $h^{(1)}(x)=h(x), A_{6}^{(1)}(x)=A_{6}(x)$, and for $k>1$, letting

$$
h^{(k+1)}(x)=\frac{1}{x} \sum_{t=1}^{x} h^{(k)}(t), \quad A_{6}^{(k+1)}(x)=\frac{1}{x} \sum_{t=1}^{x} A_{6}^{(k)}(t),
$$

it appears implausible that for any fixed $k$, we have

$$
A_{6}^{(k)}(x) \sim h^{(k)}(x) \text {. }
$$

For note that (see, e.g., [23, Section 138])

$$
\pi_{1}(x, \chi)=-\sum_{\rho} \frac{x^{\rho+1}}{\rho(\rho+1) \log x}+\mathcal{O}\left(\frac{x^{\theta}}{(\log x)^{2}}\right),
$$


where $\rho$ runs through all zeros of $L(s, \chi)$ in the critical strip and $\theta$ is the upper bound of their real parts.

If (4.19) holds and $\theta=\frac{1}{2}$ we should have

$$
\sum_{\rho} \frac{x^{\rho}}{\rho(\rho+1)^{k}}=o\left(x^{1 / 2}\right)
$$

but every term on the left-hand side of (4.21) has order $x^{1 / 2}$.

Prior to receiving Schinzel's letter, dated September 4, 1977, I had overlooked the above argument although I had noted (as did Schinzel independently) that from

$$
\lim _{k \rightarrow \infty} \sum_{\rho} \frac{1}{|\rho||\rho+1|^{k}}=0
$$

the following theorem follows at once, assuming the truth of the generalized Riemann hypothesis for $L(s, \chi)$.

TheOREM 4.2. Let $A^{(k)}(x)$ and $h^{(k)}(x)$ be defined as above. Then

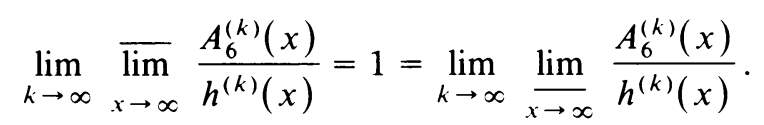

Remark. Modifications of the above arguments for $b=4$ or for $b=2$ (li $x$ versus $\pi(x))$ are easily obtained. It is, consequently, improbable that for any fixed $k \geqslant 1$, the $k$ th average of li $x-\pi(x)$ is asymptotic to the $k$ th average of li $x^{1 / 2} / 2$. It is possible that Theorem 4.1 could be generalized to rigorously prove this but $I$ have not been able to obtain this.

5. Data on $\pi_{6,5}(t)-\pi_{6,1}(t)$ and Pictorial Description of the Oscillations of $A_{6}(x)$ About $h(x)$. Our computations were carried out to $2.5 \times 10^{11}$ largely in the hope of finding the smallest integer $t$ with $\pi_{6,5}(t)<\pi_{6,1}(t)$; for the eventual success of this venture, see [4].

Figure 1 gives a value of $t$ with $\pi_{6,5}(t)-\pi_{6,1}(t)>\pi\left(t^{1 / 2}\right)$-a rare "inverted axis crossing" - and some "near axis crossings" for 2,000,000 $<t<250,000,000,000$. A "near axis crossing" is a value of $t$ with $\pi_{6,5}(t)-\pi_{6,1}(t)<1500$. The values listed in Figure 1 are pictured in Figure 4. Interestingly, they mostly occur in the vicinity of an inflection point of the graph of $A_{6}(x)$.

\begin{tabular}{crcr}
\hline Point (see Figure 4) & \multicolumn{1}{c}{$t$} & $\pi_{6,5}(t)-\pi_{6,1}(t)$ & $\pi\left(t^{1 / 2}\right)$ \\
\hline a & $344,558,471$ & 2310 & 2125 \\
b & $2,471,075,683$ & 627 & 5106 \\
c & $4,450,687,051$ & 608 & 6654 \\
d & $5,278,986,859$ & 573 & 7190 \\
e & $7,068,537,481$ & 543 & 8197 \\
f & $13,121,332,621$ & 1117 & 10834 \\
g & $24,411,171,451$ & 1193 & 14379 \\
h & $27,182,104,207$ & 730 & 15088 \\
i & $121,269,714,529$ & 1220 & 29834
\end{tabular}

FIGURE 1 


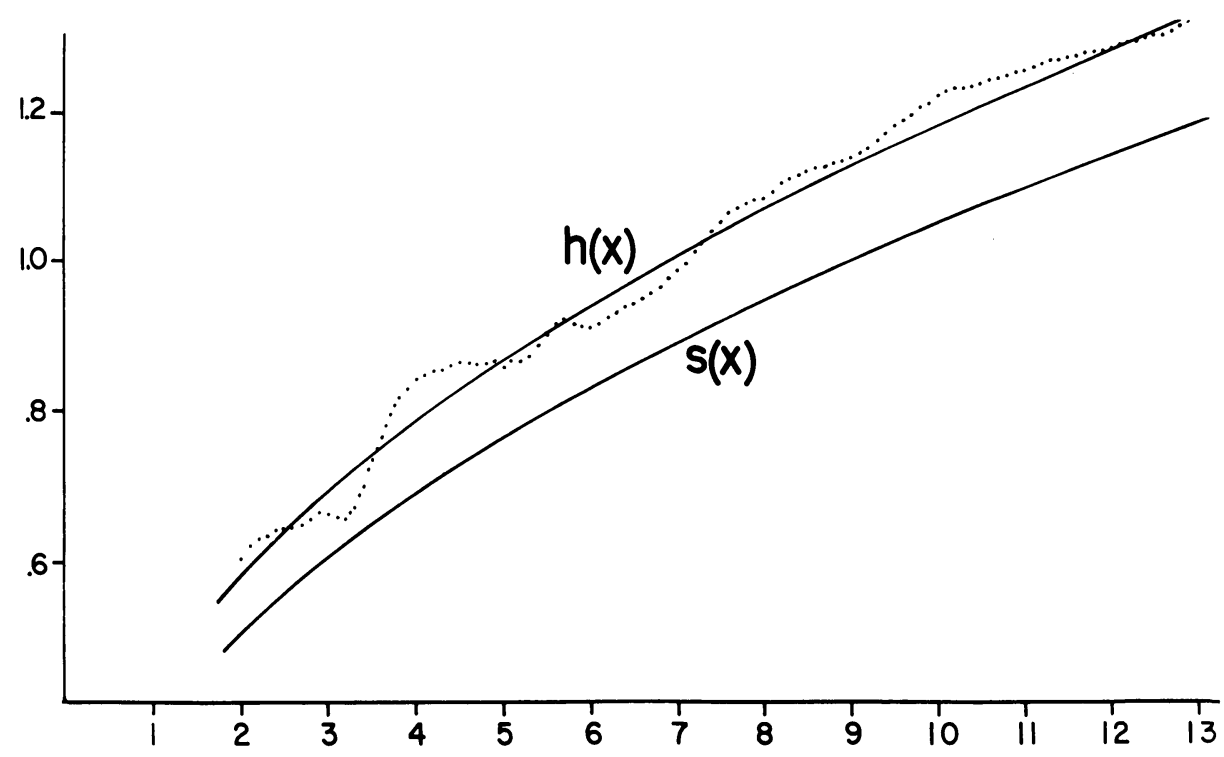

FIGURE 2

In this figure, and in Figure 3, values for $h(x), s(x)$, and $A_{6}(x)$ have been plotted on standard (increasing) scales; the dotted line represents $A_{6}(x)$. Values for $x$ are on the horizontal axis and extend from $2 \cdot 10^{8}$ to $13 \cdot 10^{8}$.

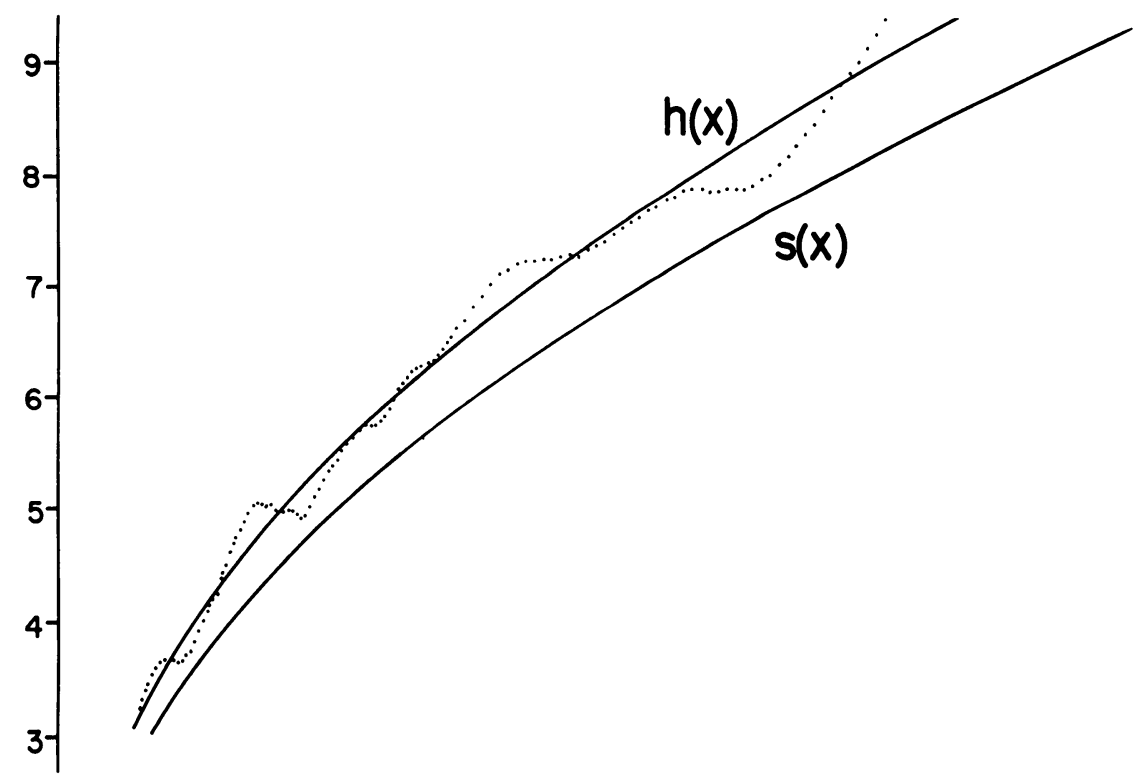

FIGURE 3

In this figure, $x$ ranges from $1 \cdot 10^{9}$ to $13 \cdot 10^{9}$. 


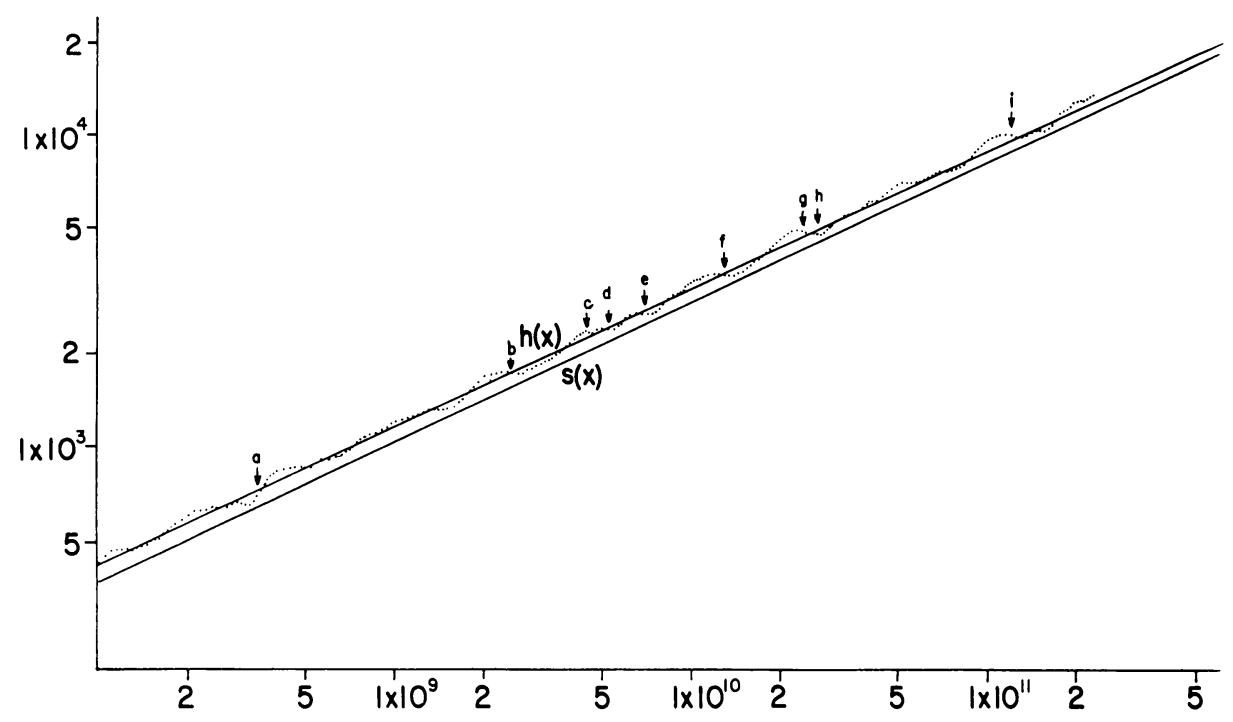

FIGURE 4

Values of $h(x), s(x)$, and $A_{6}(x)$ have been plotted on a $\log \log$ scale. Values for $x$ extend from $10^{8}$ to $2.5 \cdot 10^{11}$. The locations of points listed in Figure 1 are indicated by arrows.

Acknowledgments. I would like to thank Professors J. Ian Richards and Andrzej Schinzel for numerous helpful suggestions in the preparation of this manuscript. In particular, Schinzel's contributions in obtaining the important results in Section 4 are deeply appreciated. I would also like to thank Professor Carter Bays for assistance in preparing Figures $1, \ldots, 4$ and Professor John Leech for his constant encouragement and suggestions. Finally, I would like to thank Stephen Eure for carefully reading the manuscript and making helpful suggestions.

Department of Mathematics and Statistics

University of South Carolina

Columbia, South Carolina 29208

1. R. Ayoub, An Introduction to the Analytic Theory of Numbers, Math. Surveys, No. 10, Amer. Math. Soc., Providence, R. I, 1963.

2. Carter Bays \& Richard H. Hudson, "The segmented sieve of Eratosthenes and primes in arithmetic progressions to $10^{12}$," BIT, v. 17, 1977, pp. 121-127.

3. Carter Bays \& Richard H. Hudson, "On the fluctuations of Littlewood for primes of the form $4 n \pm 1$," Math. Comp., v. 32, 1978, pp. 281-286.

4. Carter Bays \& Richard H. Hudson, "Details of the first region of integers $x$ with $\pi_{3.2}(x)<$ $\pi_{3,1}(x)$," Math. Comp., v. 32, 1978, pp. 571-576.

5. A. Bloch \& G. Polya, "On the roots of certain algebraic equations," Proc. London Math. Soc. (2), v. 33, 1932, pp. 102-114.

6. RichaRd P. BREnt, "Irregularities in the distribution of primes and twin primes," Math. Comp., v. 29, 1975 , pp. 43-56.

7. RICHARD P. BRENT, Tables Concerning Irregularities in the Distribution of Primes and Twin Primes to $10^{11}$, Computer Centre, Australian National University, Canberra, Australia, 1975.

8. P. L. Chebyshev, "Lettre de M. le professeur Tchébychev à M. Fuss sur un nouveau théorème relatif aux nombres premiers contenus dans les formes $4 n+1$ et $4 n+3$," Bull. Classe Phys.-Mathém. Acad. Imp. des Sciences, St. Petershurg, v. 11, 1853, p. 208. 
9. W. J. Ellison, Les Nombres Premiers, Hermann, Paris, 1975.

10. Emil Grosswald, "Oscillation theorems of arithmetic functions," Trans. Amer. Math. Soc., v. 126. 1967, pp. 1-28.

11. G. H. HaRdy \& J. E. Littlewood, "Contributions to the theory of the Riemann zeta function and the theory of the distribution of primes," Acta Math., v. 41, 1917, pp. 119-196.

12. Richard H. Hudson \& Alfred Brauer, "On the exact number of primes in the arithmetic progressions $4 n \pm 1$ and $6 n \pm 1$, , J. Reine Angew. Math., v. 291, 1977, pp. 23-29.

13. RiChaRd H. HudSON, "A formula for the exact number of primes below a given bound in any arithmetic progression," Bull. Austral. Math. Soc., v. 16, 1977, pp. 67-73.

14. Richard H. HudSON \& CARTER BAys, "The mean behavior of primes in arithmetic progressions," J. Reine Angew. Math., v. 296, 1977, pp. 80-99.

15. RichaRd H. HUdSON, "A common combinatorial principle underlies Riemann's formula, the Chebyshev phenomenon, and other subtle effects in comparative prime number theory, I," J. Reine Angew. Math., v. 313, 1980, pp. 133-150.

16. A. E. Ingham, The Distribution of Prime Numbers, Stechert-Hafner, New York, 1964.

17. S. KNAPOWSKI \& P. TURÁN, "Further developments in the comparative prime-number theory I." Acta. Arith., v. 9, 1964, pp. 23-40.

18. S. KNAPOWSKI \& P. TURÁN, "Further developments in the comparative prime-number theory II, A modification of Chebyshev's assertion," Acta Arith., v. 10, 1964, pp. 293-313.

19. S. KNAPOWSKI \& P. TURÁN, "Further developments in the comparative prime-number theory III," Acta Arith., v. 11, 1965, pp. 115-127.

20. S. KNAPOWSKI \& P. TURÁN, "Further developments in the comparative prime-number theory IV," Acta Arith., v. 11, 1965, pp. 147-161.

21. S. KNAPOWSKi \& P. TURÁN, "Further developments in the comparative prime-number theory V." Acta Arith., v. 11, 1965, pp. 193-202.

22. S. KNAPOWSKI \& P. TURÁN, "Further developments in the comparative prime-number theory VI. Accumulation theorems for residue-classes representing quadratic residues mod $k$," Acta Arith., v. 12. 1966, pp. 85-96.

23. E. Landau, Handbuch der Lehre von der Verteilung der Primzahlen, Chelsea, New York, 1953.

24. E. LANDAU, "Über einige ältere Vermutungen und Behauptungen in der Primzahltheorie," Math. Z., v. 1, 1919, pp. 1-24.

25. J. E. Littlewood, "Sur la distribution des nombres premiers," C. R. Acad. Sci. Paris,v. 158, 1914, pp. 1969-1972.

26. KARL K. NORTON, “Upper bounds for $k$-th power coset representatives modulo n," Acta Arith.. v. $15,1968 / 69$, pp. 161-179.

27. G. Polya, Üher das Vorzeichen des Restgliedes im Primzahlsatz, Göttingen Nachrichten, 1930, pp. $19-27$.

28. B. Riemann, Ueber die Anzahl der Primzahlen unter einer gegebenen Grösse, Monatsbericht. Preuss. Akad. Wissens. (Berlin: 1859; 1880), pp. 671-680. [Gesammelte mathematische Werke (1892), pp. 145-155.]

29. Daniel Shanks, "Quadratic residues and the distribution of primes," Math. Comp., v. 13, 1959, pp. 272-284.

30. Daniel Shanks, "Systematic examination of Littlewood's bounds on $L(1, \chi)$," Proc. Simpos. Pure Math., vol. 24, 1973, pp. 267-283.

31. Daniel Shanks, "Bateman's constants reconsidered and the distribution of cubic residues," Math. Comp., v. 26, 1972, pp. 265-285.

32. H. M. STARK, "A problem in comparative prime number theory," Acta Arith., v. 18, 1971, pp. 311-320. 